Check for updates

Cite this: RSC Adv., 2017, 7, 33258

\title{
Highly sensitive mechanochromic photonic gel towards fast- responsive fingerprinting $\dagger$
}

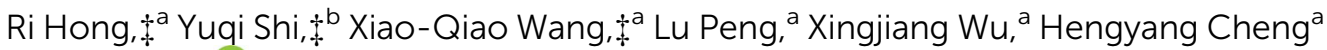 \\ and Su Chen (D) *a
}

Mechanochromic photonic materials, which are periodic structures engineered with photonic stop bands, have attracted enormous interest because of their rapid change in color upon stimulation with mechanical force. Here, we develop a high-performance mechanochromic photonic gel based on magnetically assembled, carbon-encapsulated $\mathrm{Fe}_{3} \mathrm{O}_{4}$ nanoparticles embedded into a soft copolymer of $\mathrm{N}$ hydroxymethyl acrylamide and $\mathrm{N}$-vinylcaprolactam in which the content of carbon-encapsulated $\mathrm{Fe}_{3} \mathrm{O}_{4}$ is as low as $0.18 \mathrm{wt} \%$. The photonic gel not only displays fast-dynamic and reversible color change from purple to red in a broad wavelength range $(\Delta \lambda=233 \mathrm{~nm})$, but also maintains high mechanochromic sensitivity $\left[\Delta \lambda / \sigma=53.1 \mathrm{~nm}(\mathrm{kPa})^{-1}\right]$ and spatial resolution (less than $100 \mu \mathrm{m}$ ). By taking advantage of these remarkable merits, the photonic gel has been successfully applied in the identification of color fingerprints and ultra-fine hair resolution. Considering these outstanding achievements, we believe that our photonic gel will guide the development of mechanochromic materials and open an avenue towards the design of mechanical-optical devices.

Received 18th May 2017

Accepted 15th June 2017

DOI: $10.1039 / c 7 r a 05622 b$

rsc.li/rsc-advances compressive force is applied to soft materials, the interparticle distance $d$ within the PCs will be decreased, resulting in a blue shift in the wavelength. In contrast, when a soft material is stretched, the interparticle distance will be increased, resulting in a red shift in the wavelength. After withdrawing the mechanical force, the structural color will instantly recover to its original state. Various studies have been done on the underlying mechanism of mechanochromic PCs. For example, Foulger et al. reported a mechanochromic photonic hydrogel formed by embedding a PS crystalline colloidal array into poly(ethylene glycol)/poly(2-methoxyethyl acrylate) through photo-initiated free-radical polymerization. ${ }^{30}$ The photonic colloidal PCs have great potential for many applications, including optical strain sensors, ${ }^{18-20}$ mechanical actuators, ${ }^{21}$ anti-counterfeiting applications, ${ }^{22-24}$ and tactile sensing. ${ }^{25}$

Generally, mechanochromic PCs consist of "hard" building blocks such as silica $\left(\mathrm{SiO}_{2}\right){ }^{26}$ polystyrene $(\mathrm{PS})^{27}$ and carbonencapsulated $\mathrm{Fe}_{3} \mathrm{O}_{4}$ (ref. 28) interpenetrated with soft materials such as gels and rubbers. ${ }^{29}$ Scheme 1 shows the mechanochromic mechanism of PCs. When a mechanical

${ }^{a}$ State Key Laboratory of Materials-Oriented Chemical Engineering, College of Chemical Engineering, Jiangsu Key Laboratory of Fine Chemicals and Functional Polymer Materials, Nanjing Tech University (Former: Nanjing University of Technology), Nanjing 210009, P. R. China.E-mail: chensu@njtech.edu.cn

${ }^{b}$ School of Chemical and Material Engineering, Fuyang Normal University, Fuyang 236001, P. R. China

$\dagger$ Electronic supplementary information (ESI) available. See DOI: 10.1039/c7ra05622b

\$ Contribute equally to this work.
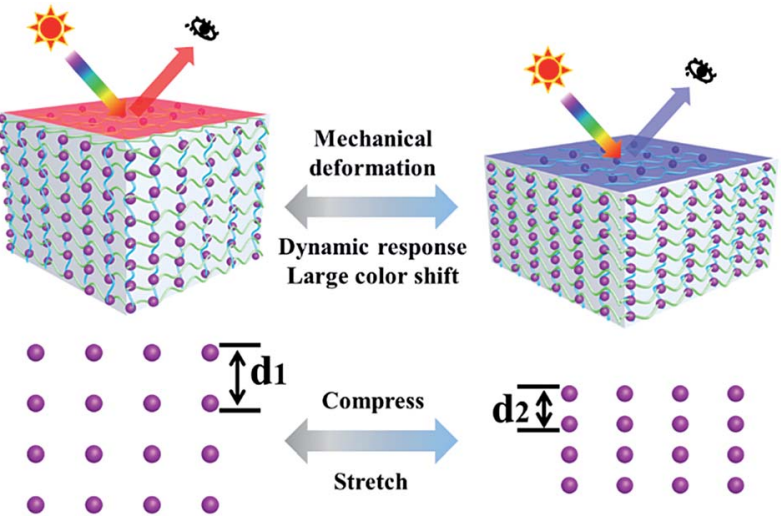

Scheme 1 Schematic illustration of the mechanochromic mechanism of a PC gel. 
hydrogel displayed an excellent blue shift $(\Delta \lambda=93 \mathrm{~nm})$ and stress sensitivity $\left[\Delta \lambda / \sigma=0.64 \mathrm{~nm} \cdot(\mathrm{kPa})^{-1}\right]$. Arsenault's group developed a porous inverse photonic film based on $\mathrm{SiO}_{2}$ nanoparticles that demonstrated enhanced mechanical sensitivity $\left[4 \mathrm{~nm}(\mathrm{kPa})^{-1}\right]$ as well as fast response speed (several seconds). ${ }^{31}$ Zhu et al. utilized magnetic nanoparticles combined with acrylamide to fabricate a mechanochromic photonic hydrogel with high sensitivity $\left[46.5 \mathrm{~nm}(\mathrm{kPa})^{-1}\right]$ and mechanical stability (150 cycles). ${ }^{32}$ A photonic gel based on a lamellar structure was designed and exhibited a large blue shift $(200 \mathrm{~nm})$ in response to strain. ${ }^{33}$ Moreover, a block copolymer (polystyrene- $b$-poly-2vinylpyridine) photonic gel was also employed with promoting the mechanical sensitivity, which revealed tremendous blue shift $(240 \mathrm{~nm}){ }^{34}$ Obviously, mechanochromic PCs perform outstanding optical properties during the process of mechanical force. However, the relatively slow-dynamic reversible color variation, low mechanochromic sensitivity and poor spatial resolution severely discourage their further optical application.

In this work, we report an effective one-step strategy to fabricate a high-performance mechanochromic PC gel through magnetic assembly combined with the fast free-radical polymerization of $\mathrm{Fe}_{3} \mathrm{O}_{4}$ within $N$-hydroxymethyl acrylamide (NMA) and $\mathrm{N}$-vinylcaprolactam (VCL). To maintain the outstanding intrinsic flexibility of the gel, the "hard" content of $\mathrm{Fe}_{3} \mathrm{O}_{4}$ colloidal particles was manipulated to be as low as $0.18 \mathrm{wt} \%$ whereas the vividly structural color can be still observed. By utilizing various molds, cylindrical, sliced and film-like photonic gels are obtained. The photonic gel exhibits fast dynamic red-shifting color from 407 to $640 \mathrm{~nm}$ under $13.4 \mathrm{kPa}$ of compressive stress. The photonic gel also exhibited large stress sensitivity $\left[53.1 \mathrm{~nm}(\mathrm{kPa})^{-1}\right]$, mechanochromic strain sensitivity (5.75 $\mathrm{nm}$ per \%), high spatial resolution $(100 \mu \mathrm{m})$ and good reversible durability. Underlying the excellent properties of photonic gels, we have realized ultra-fine hair identification and color fingerprint upon compression-relaxation. Our work clarifies the important role of high sensitivity and spatial resolution design, which will promote the development of nextgeneration optical applications.

\section{Results and discussion}

The preparation process of the PC gel is schematically illustrated in Fig. 1. A mixture of carbon-encapsulated magnetic $\mathrm{Fe}_{3} \mathrm{O}_{4}$ colloidal particles, NMA, VCL, the redox couple benzoyl
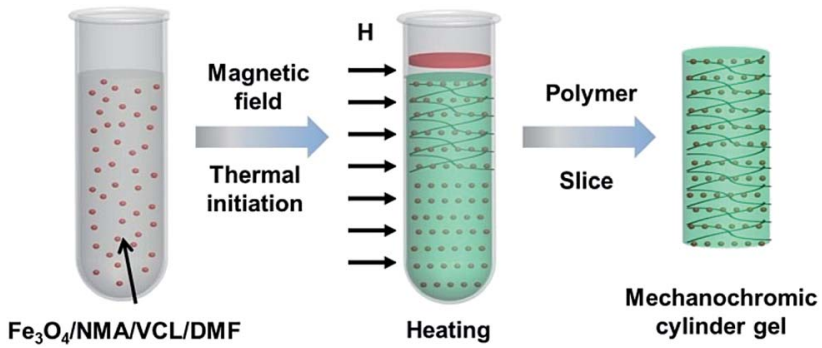

Fig. 1 Schematic preparation of the cylindrical PC gel. peroxide (BPO) $/ N, N$-dimethylaniline (DMA) and $N$-dimethylformamide (DMF) was uniformly dispersed in a glass tube. A brilliant structural color was observed when the magnetic field was applied perpendicularly to the glass tube, indicating that the $\mathrm{Fe}_{3} \mathrm{O}_{4}$ particles were directionally arranged to form 1D photonic chains along the magnetic field. ${ }^{35}$ Simultaneously, radical polymerization was ignited from the upper layer of the mixture. After 2 min of polymerization, we obtained a cylindrical PC gel. The entire fabrication process of the photonic gel could be rapidly completed within several minutes, demonstrating the higher efficiency of this method compared to conventional methods.

The microscopic morphology of the photonic gel was characterized by scanning electron microscopy (SEM) and transmission electron microscopy (TEM). Fig. 2a shows a typical TEM image of the magnetic $\mathrm{Fe}_{3} \mathrm{O}_{4}$ particles with an average size of $c a$. $100 \mathrm{~nm}$. Through compositional modulations, various sizes (90, 140 and $180 \mathrm{~nm}$ ) of $\mathrm{Fe}_{3} \mathrm{O}_{4}$ were realized (Fig. S1 $\dagger$ ). The arrangement of $\mathrm{Fe}_{3} \mathrm{O}_{4}$ particles in the gel is illustrated in Fig. $2 \mathrm{~b}$. Notably, the $\mathrm{Fe}_{3} \mathrm{O}_{4}$ particles were aligned to form 1D photonic chains inside the gel, demonstrating the effective magneticfield induction. Changing the sizes of $\mathrm{Fe}_{3} \mathrm{O}_{4}$ resulted in tunable structural colors of the photonic gels. The related multicolor photographs were purple, blue, cyan, green and orange-red (Fig. 2c), and their corresponding reflection peaks
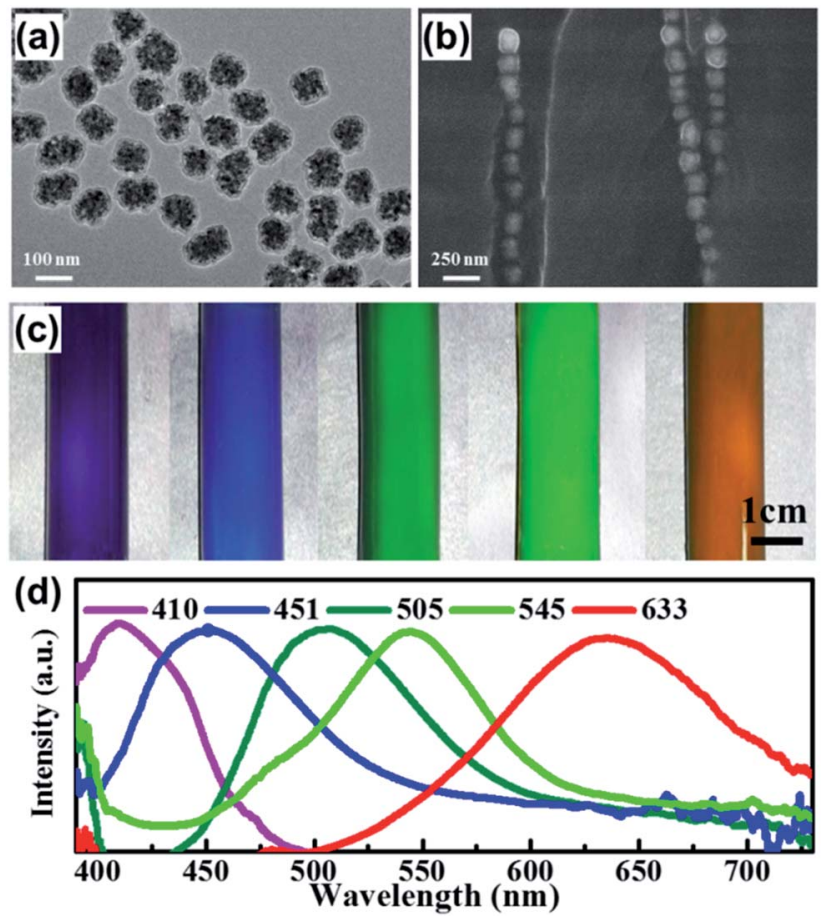

Fig. 2 Morphology and reflection spectra characterization of the photonic gel. (a) TEM image of typical $\mathrm{Fe}_{3} \mathrm{O}_{4}$ colloidal particles. (b) Cross-sectional SEM image of the $1 \mathrm{D} \mathrm{Fe} \mathrm{O}_{4} \mathrm{PC}$ arrangement in the gel after magnetic-field induction. (c) Photographs of purple, blue, cyan, green and orange red cylindrical photonic gels. (d) The corresponding reflection spectra measured by an optical microscope equipped with a fiber optic spectrometer of cylindrical photonic gels in (c), the reflection peaks assigned at 410,451, 505, 545 and $633 \mathrm{~nm}$. 
with stop bands were assigned at 410, 451, 505, 545 and 633 $\mathrm{mm}$, respectively (Fig. 2d). Concomitantly, photonic gels with different shapes (cylindrical, sliced and heart-shaped) could be prepared by using different molds (Fig. S2†).

The photonic gel exhibited high sensitivity to compressive force along with fast mechanochromic response. Fig. 3a and b show the color change and their respective reflection spectra for gels under different compressive strains $(\varepsilon)$. The colors and reflection peaks were shifted from the purple to the red wavelength region with increasing compressive strain, indicating full color mechanochromy. Furthermore, the minimum strain generating a red shift (peak wavelength from 407 to $476 \mathrm{~nm}$, $\Delta \lambda_{\min }=69 \mathrm{~nm}$ ) was $12 \%$. The maximum strain producing a red shift (peak wavelength from 407 to $640 \mathrm{~nm}, \Delta \lambda_{\max }=233 \mathrm{~nm}$ ) was $49 \%$. The red shift in the colors of the cylindrical gels is attributed to the applied compressive force in the axial direction, which stretched the 1D photonic chains in the radial direction. Fig. $3 \mathrm{c}$ illustrates the detailed strain $(\varepsilon)$-dependent mechanochromic sensitivity $(\Delta \lambda / \Delta \varepsilon)$ and compressive pressure $(\sigma)$. It is revealed that $\Delta \lambda / \Delta \varepsilon$ decreased with increasing $\varepsilon$, and the maximum was $5.75 \mathrm{~nm}$ per \%. Additionally, when the compressive stress $(\sigma)$ increased from 0 to $13.4 \mathrm{kPa}$, the gel color shifted from purple to red. We further calculated the stress sensitivity behaviors of the photonic gels. The maximum stress sensitivity $\left(\Delta \lambda_{\min } / \sigma\right)$ was $53.1 \mathrm{~nm}(\mathrm{kPa})^{-1}$ at the compressive stress of $1.3 \mathrm{kPa}$. To the best of our knowledge, the mechanochromic sensitivity of our photonic gel is one of the highest reported for colloidal crystals. ${ }^{30,31,36}$

The fast-dynamic mechanochromic properties of the photonic gel were investigated by driving the gel under compression-relaxations process. As shown in Fig. 4a, the brilliant blue gel underwent dynamically reversible color changes in response to mechanical compression-relaxation, particularly a multicolor variation from blue to cyan, green,

(a)
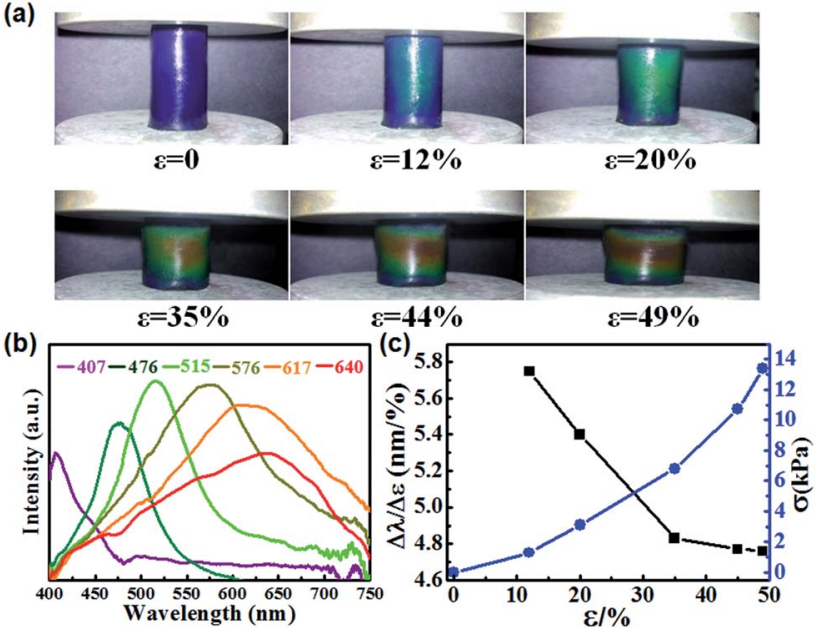

Fig. 3 Highly sensitive full-color mechanochromy of photonic gels. (a) Photographs and (b) reflection spectra of the cylindrical photonic gel under different compressive strains $(\varepsilon)$. (c) Compressive strain $(\varepsilon)$ dependent mechanochromic sensitivity $(\Delta \lambda / \Delta \varepsilon)$ and compressive stress $(\sigma)$.
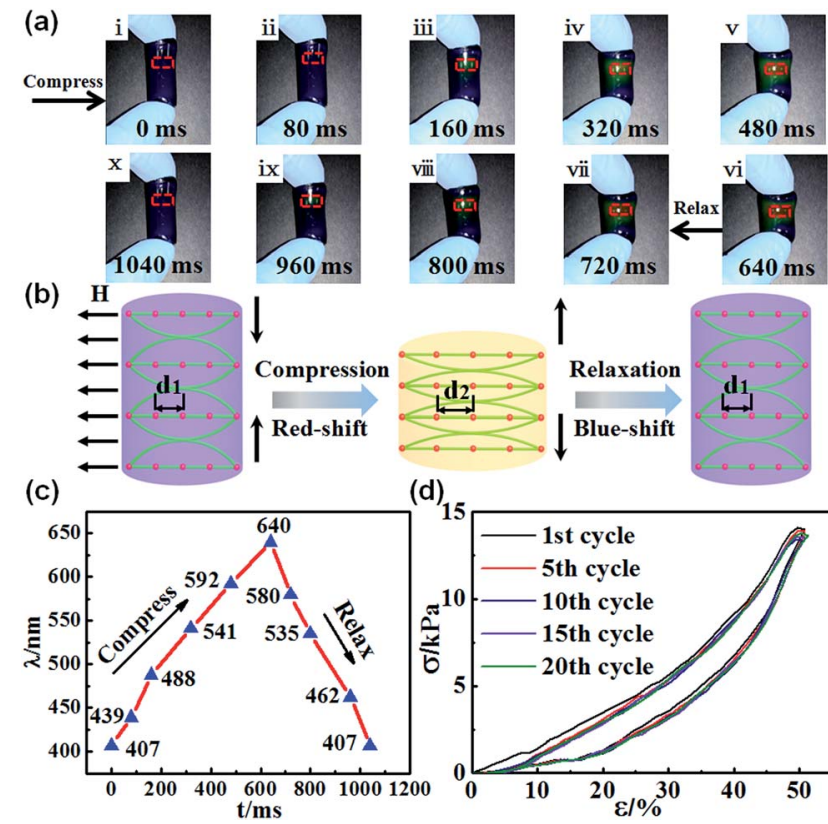

Fig. 4 Fast-reversible color switching of photonic gels. (a) Images of a photonic gel during a compression-relaxation process. (b) Schematic illustration of the photonic gel mechanochromic mechanism during the reversible process. (c) Relationship between reflection wavelength and response time. (d) Stress-strain curves of the gel during cycling tests.

orange and red in the center of the gel. Meanwhile, an iridescent color gradient existed in the gel because of the gradient stress field. The excellent reversibility of the color changes was ascribed to the elastic property, which made the gel sufficiently robust to withstand rapid mechanical force. The mechanism explaining the fast-reversible color switching of the photonic gel is described in Fig. 4b. When mechanical compressive force was applied to the gel, the horizontal one-dimensional (1D) photonic chains embedded in the copolymer gel spread. As a result, the interparticle distance $d$ increased, bringing about the red shift in the reflection wavelength. ${ }^{28,32}$ In contrast, upon withdrawing the mechanical compression, the interparticle distance $d$ recovered to its initial state, and the structural color was blue-shifted. We further measured the reflection spectrum using an optical microscope equipped with a fiber-optic spectrometer. The optical fiber probe was perpendicular to the region of color change (red dotted area in Fig. 4a). Concomitantly, the variation in the reflection spectrum with response time is illustrated in Fig. 4c. The corresponding response time for red-shifting (407-640 nm) compression was about $640 \mathrm{~ms}$, and that for recovering to the original state was about $400 \mathrm{~ms}$. We further tested the compression-relaxation durability of the photonic gel under a strain level of 50\% (Fig. 4d). Obviously, the photonic gel presented good cyclic stability without any notable decline after continuous operation for 20 cycles. Movie S1 $\dagger$ shows the entire process of deformation and reversible color switching for the photonic gel. The photonic gel displayed high mechanochromic sensitivity and fast reversible color switching, ensuring superior performance in subsequent applications. 
To explore the potential application of the mechanochromic gel in displays, a flexible photonic gel thin film was fabricated. As depicted in Fig. 5a, the photonic gel film was prepared by the thermal polymerization of mixture under the magnetic field in the reactor. The height of reactor is $\sim 0.5 \mathrm{~cm}$. Green and red photonic gel films (Fig. S3†) that could bend flexibly (Fig. S4 †) were fabricated. Ten casual points were selected from the green gel, and the corresponding reflection wavelengths $(\sim 519 \mathrm{~nm})$ demonstrated the uniformity of the gel film (Fig. S5†). To investigate the spatial resolution of the photonic gel film, an ultra-fine hair (diameter $=100 \mu \mathrm{m}$ ) was introduced to evaluate the performance. As seen in Fig. 5b, an evident color change from green to red-orange occurred when the hair was removed from the surface of the film, as demonstrated by the shift in reflection wavelength from 519 to $653 \mathrm{~nm}$ (Fig. 5c). Movie S2 $\dagger$ recorded the high spatial resolution of gel film to respond the ultra-fine hair. After taking full use of the high spatial resolution, a significant application was conducted by identifying the tiny and fine fingerprint. As shown in Fig. 5d, when an index finger was removed from the gel film, a clear color fingerprint image was stamped on the gel surface, and the image gradually disappeared in about ten seconds. Movie S3 $\uparrow$ presents the colorchange process of fingerprinting in response to finger compression and release. Fig. 5e illustrates the mechanism of fingerprint identification. When the index finger was removed from the gel surface, the contacted finger stretched the photonic gel. Under this condition, the $\mathrm{Fe}_{3} \mathrm{O}_{4}$ particle distance
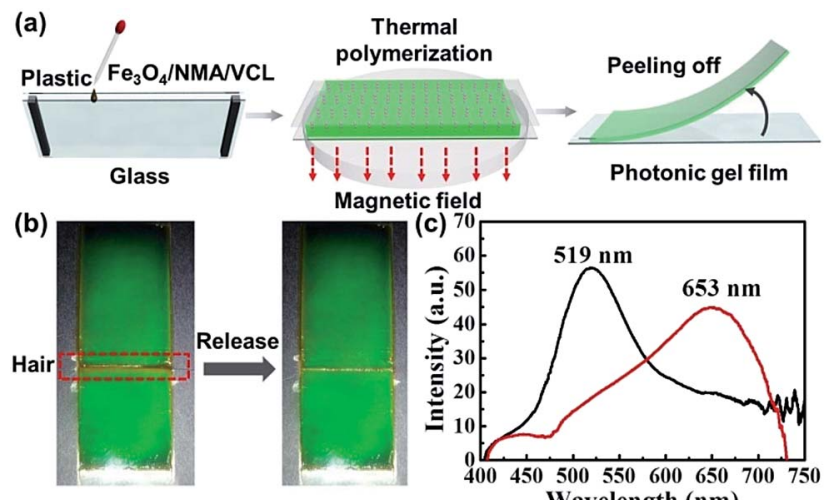

(d)
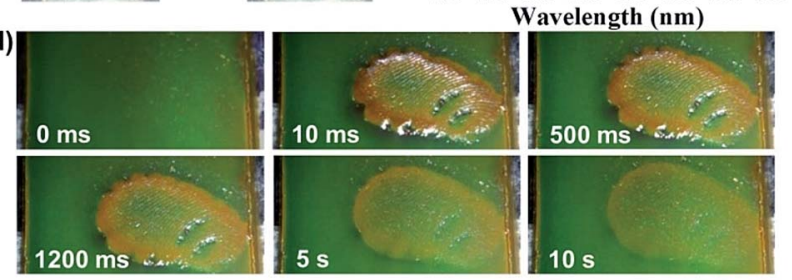

(e)

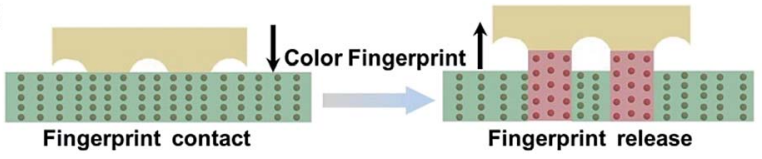

Fig. 5 Mechanochromic fingerprinting using the photonic gel. (a) Schematic of the preparation of a flexible photonic gel film. Photographs (b) and reflection spectra (c) of the gel film before and after monitoring human hair. (d) Photographs recording the color-fingerprinting process. (e) Schematic mechanism of fingerprint identification. increased, resulting the red shift of the wavelength and thus the appearance of a color image on the gel film. Based on the unique lines of human fingers, the fingerprint could be identified.

\section{Conclusions}

In summary, we demonstrated a novel mechanochromic PC gel formed via the fast polymerization of magnetically assembled $\mathrm{Fe}_{3} \mathrm{O}_{4}$. Colorful and shape-moldable gels including cylindrical, sliced and film-like PC gels were obtained. The photonic gels displayed brilliant structural color, even at $\mathrm{Fe}_{3} \mathrm{O}_{4}$ particle contents as low as $0.18 \mathrm{wt} \%$. The cylindrical photonic gels exhibited fast-dynamic and reversible color variation (from 407 to $640 \mathrm{~nm})$, high mechanochromic sensitivity $[\Delta \lambda / \sigma=53.1 \mathrm{~nm}$ $(\mathrm{kPa})^{-1}$ and $\Delta \lambda / \Delta \varepsilon=5.75 \mathrm{~nm}$ per \%] and spatial resolution (less than $100 \mu \mathrm{m}$ ). The performance of the photonic gel is one of the best reported in the literature to date. Based on its outstanding performance, the gel was successfully applied in color fingerprint identification and ultra-fine hair resolution. The gels show great potential for various applications including fast sensors, high-precision displays and new fingerprinting materials.

\section{Experimental}

\section{Materials}

Ferrocene $\left[\mathrm{Fe}\left(\mathrm{C}_{5} \mathrm{H}_{5}\right)_{2},>98 \%\right]$, hydrogen peroxide $\left(\mathrm{H}_{2} \mathrm{O}_{2}, 30 \%\right)$, acetone $\left(\mathrm{C}_{3} \mathrm{H}_{6} \mathrm{O},>99 \%\right)$, NMA, VCL, $N, N^{\prime}$-methylene-bisacrylamide (MBA), BPO/DMA (redox couple), and DMF were purchased from Aldrich.

\section{Preparation of colloidal PC gel}

Carbon-encapsulated $\mathrm{Fe}_{3} \mathrm{O}_{4}$ particles $(0.05 \mathrm{~g})$ were dispersed in $10 \mathrm{~g}$ DMF and sonicated until a homogeneous solution was formed. Then, $2.16 \mathrm{~g}$ NMA, $1.44 \mathrm{~g}$ NVC and $0.005 \mathrm{~g}$ MBAA were added in $2.0 \mathrm{~g}$ as-prepared above homogeneous solution (the dispersion of carbon-encapsulated $\mathrm{Fe}_{3} \mathrm{O}_{4}$ in DMF) and intensely sonicated to obtain a homogeneous mixture. Subsequently, $0.016 \mathrm{~g}$ BPO and $0.016 \mathrm{~g}$ DMA were added to the mixture and shaken vigorously for seconds. To prepare cylindrical gels with structural color in the axial direction, the mixture was transferred into a test tube with diameter of $12 \mathrm{~mm}$ that was fixed parallel on the center of an NdFeB magnet with a magnetic field of about $700 \mathrm{G}$ perpendicular to the tube. The upper layer of the mixture was triggered by a soldering iron at $100{ }^{\circ} \mathrm{C}$, and cylindrical photonic gels were obtained after several minutes of thermo-polymerization. To prepare gels with purple, blue, cyan, green and red-orange colors, carbon-encapsulated $\mathrm{Fe}_{3} \mathrm{O}_{4}$ particles with various sizes in the range of 90-180 nm were also fabricated. To prepare sliced gels with color in the radial direction, the orientation of the applied magnetic field was changed to be along the tube filled with polymer precursor before thermo-polymerization. To prepare photonic gel films, $2.16 \mathrm{~g}$ NMA, $1.44 \mathrm{~g}$ VCL and $0.0025 \mathrm{~g}$ MBAA were ultrasonically dispersed in $2.0 \mathrm{~g}$ as-prepared solution containing carbonencapsulated $\mathrm{Fe}_{3} \mathrm{O}_{4}$ particles. Next, $0.03 \mathrm{~g} \mathrm{BPO}$ and $0.03 \mathrm{~g}$ 
DMA were added to the mixture and shaken vigorously for seconds. The mixture was injected into the space between a glass substrate and a transparent plastic film $(25 \mathrm{~mm} \times 60$ $\mathrm{mm} \times 5 \mathrm{~mm}$ ) that was isolated by adhesive tape. The device was then placed on the center of the $\mathrm{NdFeB}$ magnet for thermopolymerization. Finally, the photonic film on the clear plastic film could be easily peeled off from the glass substrate.

\section{Characterization}

The morphologies of the $\mathrm{Fe}_{3} \mathrm{O}_{4}$ particles were characterized by TEM with an accelerating voltage of $200 \mathrm{kV}$ (JEOL-2100). The diameters of the $\mathrm{Fe}_{3} \mathrm{O}_{4}$ particles and 1D photonic chains composed of $\mathrm{Fe}_{3} \mathrm{O}_{4}$ particles in the photonic gel were examined by SEM using a QUANTA200 (Philips-FEI, Holland) instrument at $30.0 \mathrm{kV}$. Stress-strain tests were performed on the cylindrical gel samples $(D=12 \mathrm{~mm})$ using a SANS CMT6203 testing machine at $25{ }^{\circ} \mathrm{C}$. In the cyclic compressive stress-strain tests, the gels were compressed to a fixed strain of $50 \%$ and then immediately relaxed to zero strain at a loading/unloading speed of $2 \mathrm{~mm} \mathrm{~s}^{-1}$. The process was cycled 20 times. The magnetic strength of the magnetic assembly of $\mathrm{Fe}_{3} \mathrm{O}_{4}$ particles during the fabrication process of the photonic gels was measured by a MZ201 teslameter. The reflective spectra were measured by an optical microscope equipped with a fiber-optic spectrometer (Ocean Optics, USB 4000) in the range of $400-700 \mathrm{~cm}^{-1}$. The dynamic color-change processes as well as the images of the prepared photonic gels were recorded by a Nikon digital camera D3100.

\section{Acknowledgements}

This work was supported by the National Natural Science Foundation of China (21474052), National Key Research and Development Program of China (2016YFB0401700) and Priority Academic Program Development of Jiangsu Higher Education Institutions (PAPD).

\section{Notes and references}

1 J. H. Holtz and S. A. Asher, Nature, 1997, 389, 829-832.

2 J. P. Ge and Y. D. Yin, Angew. Chem., Int. Ed., 2011, 50, 14921522.

3 C. I. Aguirre, E. Reguera and A. Stein, Adv. Funct. Mater., 2010, 20, 2565-2578.

4 K. Matsubara, M. Watanabe and Y. Takeoka, Angew. Chem., Int. Ed., 2007, 46, 1688-1692.

5 S. N. Yin, C. F. Wang, S. S. Liu and S. Chen, J. Mater. Chem. C, 2013, 1, 4685-4690.

6 C. Mille, E. C. Tyrode and R. W. Corkery, $R S C A d v ., 2013,3$, 3109-3117.

7 X. Q. Wang, S. Y. Yang, C. F. Wang, L. Chen and S. Chen, Macromol. Rapid Commun., 2016, 37, 759-768.

8 F. Y. Yan and S. Asher, Anal. Bioanal. Chem., 2007, 387, 21212130.

9 H. Xuan, J. Ren, Y. Zhu, B. Zhao and L. Ge, RSC Adv., 2016, 6, 36827-36833.
10 H. Xu, P. Wu, C. Zhu, A. Elbaz and Z. Z. Gu, J. Mater. Chem. C, 2013, 1, 6087-6098.

11 L. Bai, Z. Y. Xie, K. D. Cao, Y. J. Zhao, H. Xu, C. Zhu, Z. D. Mu, Q. F. Zhong and Z. Z. Gu, Nanoscale, 2014, 6, 5680-5685.

12 H. Shafiee, E. A. Lidstone, M. Jahangir, F. Inci, E. Hanhauser, T. J. Henrich, D. R. Kuritzkes, B. T. Cunningham and U. Demirci, Sci. Rep., 2014, 4, 4116.

13 Z. Y. Cai, N. L. Smith, J. T. Zhang and S. A. Asher, Anal. Chem., 2015, 87, 5013-5025.

14 L. L. Duan, B. You, L. M. Wu and M. Chen, J. Colloid Interface Sci., 2011, 353, 163-168.

15 M. Kolle, A. Lethbridge, M. Kreysing, J. J. Baumberg, J. Aizenberg and P. Vukusic, Adv. Mater., 2013, 25, 2239-2245.

16 Z. Y. Ma, Z. J. Wang, Z. J. Xu, X. R. Jia and Y. Wei, J. Mater. Chem. C, 2015, 3, 3399-3405.

17 E. P. Chan, J. J. Walish, A. M. Urbas and E. L. Thomas, Adv. Mater., 2013, 25, 3934-3947.

18 S. Liu, K. M. Yang, Y. P. Wang, J. L. Qu, C. R. Liao, J. He, Z. Y. Li, G. L. Yin, B. Sun, J. T. Zhou, G. J. Wang, J. Tang and J. Zhao, Sci. Rep., 2015, 5, 7624.

19 L. M. Fortes, M. C. Goncalves and R. M. Almeida, Opt. Mater., 2011, 33, 408-412.

20 M. A. Haque, G. Kamita, T. Kurokawa, K. Tsujii and J. P. Gong, Adv. Mater., 2010, 22, 5110-5114.

21 W. Park and J. B. Lee, Appl. Phys. Lett., 2004, 85, 4845-4847. 22 H. Nam, K. Song, D. Ha and T. Kim, Sci. Rep., 2016, 6, 30885. 23 T. Ding, G. S. Cao, C. G. Schaefer, Q. B. Zhao, M. Gallei, S. K. Smoukov and J. J. Baumberg, ACS Appl. Mater. Interfaces, 2015, 7, 13497-13502.

24 S. Y. Ye, Q. Q. Fu and J. P. Ge, Adv. Funct. Mater., 2014, 24, 6430-6438.

25 X. Q. Wang, C. F. Wang, Z. F. Zhou and S. Chen, Adv. Opt. Mater., 2014, 2, 652-662.

26 J. M. Jethmalani and W. T. Ford, Chem. Mater., 1996, 8, 21382146.

27 S. H. Foulger, P. Jiang, A. C. Lattam, D. W. Smith and J. Ballato, Langmuir, 2001, 17, 6023-6026.

28 J. P. Ge and Y. D. Yin, Adv. Mater., 2008, 20, 3485-3491.

29 Y. F. Yue, T. Kurokawa, M. A. Haque, T. Nakajima, T. Nonoyama, X. F. Li, I. Kajiwara and J. P. Gong, Nat. Commun., 2014, 5, 4659.

30 S. H. Foulger, P. Jiang, Y. R. Ying, A. C. Lattam, D. W. Smith and J. Ballato, Adv. Mater., 2001, 13, 1898.

31 A. C. Arsenault, T. J. Clark, G. Von Freymann, L. Cademartiri, R. Sapienza, J. Bertolotti, E. Vekris, S. Wong, V. Kitaev, I. Manners, R. Z. Wang, S. John, D. Wiersma and G. A. Ozin, Nat. Mater., 2006, 5, 179-184.

32 X. L. Jia, J. Y. Wang, K. Wang and J. T. Zhu, Langmuir, 2015, 31, 8732-8737.

33 Y. F. Yue, M. A. Haque, T. Kurokawa, T. Nakajima and J. P. Gong, Adv. Mater., 2013, 25, 3106-3110.

34 E. P. Chan, J. J. Walish, E. L. Thomas and C. M. Stafford, Adv. Mater., 2011, 23, 4702-4706.

35 L. Zhuang, Y. Zhao, H. Zhong, J. Liang, J. Zhou and H. Shen, Sci. Rep., 2015, 5, 17063.

36 D. Yang, S. Ye and J. Ge, Adv. Funct. Mater., 2014, 24, 31973205. 